\title{
HISTORIA A DEBATE. ACTAS DEL CONGRESO INTERNACIONAL «A HISTORIA A DEBATE» (3 Vol.) SANTIAGO 1995
}

\author{
Por \\ JOSÉ LUIS ÁLVAREZ FERNÁNDEZ*
}

En las coyunturas de ciencia normal (Kuhn) los miembros de una comunidad científica no se manifiestan en los términos de Wolfang Pauli a la altura de 1924: «En este momento la física se encuentra en un estado de terrible confusión. De cualquier modo, me resulta demasiado difícil y me gustaría haber sido actor de cine o algo por el estilo, y no haber oído hablar nunca de la física». Durante décadas, nuestra confiada inmersión en la objetivista-estructuralista historia económico-social ni nos había hecho suponer que algún día diríamos -o al menos pensaríamos- algo similar a las sinceras palabras de Pauli, esa profunda expresión de un paradigma en crisis (de nuevo, Kuhn). Y es que hoy, rodeados por la tupida maraña de los discursos sobre el fin de la historia, el giro lingüístico o el retorno de viejos géneros historiográficos, tal vez los historiadores podrán decirpensar como el Pauli de 1924. Sin embargo, conviene recordar que a la zozobra del físico austríaco le sigue de inmediato su enunciado del principio de exclusión (1925), para más tarde formular su hipótesis acerca del neutrino,... y aún tiene tiempo para degustar el protocolo de la monarquía sueca: obtiene el Premio Nobel de Física en 1945.

\footnotetext{
* Universidad de Santiago.

"CUADERNOS DE ESTUdiOS GALLEGOS", Tomo XLV, Fascículo 110, Santiago 1998.
} 
Al igual que Pauli, no parece que los historiadores -en-crisis tengan serias intenciones de volcarse hacia el séptimo arte, más bien parecen persistir en un empecinamiento parejo al de nuestro Premio Nobel: sin física teórica extraordinaria no es posible acceder a una física experimental normal, sin profundización en la teoría de la historia no se podrá redefinir permanentemente el territorio del historiador. Así, la Convocatoria del Congreso Internacional Historia A Debate (Santiago de Compostela, 7 al 11 de julio de 1993) proclama: «queremos, desbordando todo minifundismo intelectual, fomentar y poner al día el intercambio historiográfico desde la pluralidad. Nos contentaríamos, pues, con el mínimo necesario: delimitar problemas, informar de alternativas, encauzar debates, conocer planteamientos recientes, mostrar, en suma, cómo pese a todo la historia continúa, lucha, se renueva». Por consiguiente, los historiadores persisten en los Combates por la Historia (Lucien Febvre), contestan a los embates venidos desde otros territorios del conocimiento, debaten sobre anomalías surgidas en el propio territorio del conocimiento histórico, redefinen su función social. Momento, pues, de fuerte y enriquecedor debate teórico, ocasión para comenzar a reconstruir el paradigma común de los historiadores en el pasar página al siglo-milenio, tal parece ser el norte-guía de Historia A Debate [HAD].

Debido a la cantidad-densidad de contenidos de HAD, en el espacio de una breve reseña se hace imposible cubrir el pormenorizado análisis de todas y cada una de las aportaciones contenidas en las Actas [y aún informa el editor sobre la publicación de actas temáticas dedicadas a las aportaciones de medievalistas, historiadores gallegos e historiadores latinoamericanos]. De ahí que optemos por intentar ofrecer una panorámica general, dejándonos guiar por el propio Índice de las Actas, para, eventualmente, incidir en la identificación de las líneas-fuerza que hayan podido orientar la aparente heterogeneidad del material que en ellas se nos ofrece, tarea que como valoración de conjunto abordamos en las conclusiones finales. Es más, frente a la muy extendida interpretación del actual hacer historiográfico como un big-bang atomizador, como un desmigajamiento (François Dosse) del discurso histórico, urge trabajar en orientaciones, en mapas que registren la coherencia en las nuevas formas de escribir la historia.

En primer lugar, y como ya hemos adelantado, HAD constata que los historiadores no parecen estar dispuestos a ser sujetos pacientes, anodi-

"CUADERNOS DE ESTUDIOS GALLEGOS", Tomo XLV, Fascículo 110, Santiago 1998. 
nos receptores de gran teoría ordenadora o planificadora externa de la producción historiográfica. Institucionalización académica, proliferación de congresos, enriquecimiento del debate en publicaciones periódicas especializadas o diálogo crítico con las Ciencias Sociales, es decir, la red de caracteres profesionales que han ido constituyendo a la comunidad de los historiadores, todo ello ha contribuido a gestar en el historiador el hábito de diagnóstico sobre su propia producción. Tal es la orientación general que percibimos en PASADO Y FUTURO, Tomo I de las Actas. Este énfasis polémico, no contemporizador, contestatario, asoma desde las primeras páginas, desde las mismas PRESENTACIÓN y CONVOCATORIA del Congreso, extendiéndose de inmediato hacia los RESÚMENES DE LAS INTERVENCIONES DE LOS PONENTES en las MESAS REDONDAS: Final de la Historia [Paul Freedman, Carlos Martínez Shaw, Lawrence Stone]; Marxismo e Historia de los Años 90 [Chris Wickham, Bolívar Echeverría, Alain Guerreau, Juan Trías Vejarano]; ¿Crisis de la Historia Económica y Social? [Giovanni Levi, Julián Casanova, Jean-Yves Grenier, Alejandro Tortolero Villaseñor]; El «Tournant Critique» de 'Annales' [Bernard Lepetit, Carlos Aguirre Rojas, Carlos Barros, Yuri L. Bessmertny, Peter Burke]; Retorno del Sujeto [Eloy Benito Ruano]; La Historia ante las Revoluciones del Este [Marta Bizcarrondo, Robert Darnton]; La Construcción de Europa desde la Historia [Chris Wickham, Karl Friedrich Rudolf]; Revisionismo Historiográfico [Perry Anderson, David Ruiz, Gabrielle M. Spiegel]; La Historia entre la Ciencia y la Vulgarización [Alain Boureau, Antonio Simôes Rodrigues]; Historia, Investigación y Política Científica [Javier Faci]; La Historia en las Universidades [José Luis Martín, Nikolai Kopossov, Teófilo F. Ruiz, Bernard Vincent]; La Historia en las Enseñanzas Medias [Julio Valdeón, Antonio Simôes Rodrigues]; La Historiografía en España, Hoy [Julio Aróstegui, José Luis de la Granja, Julio Valdeón, Bernard Vincent].

Las resumidas intervenciones de los ponentes, en las Mesas Temáticas servirán al lector como primera aproximación a los contenidos que a continuación, y mas pormenorizadamente, se extienden a lo largo de los tres volúmenes. [De la Mesa B, Marxismo e Historia en los Años 90, se transcriben las intervenciones de los miembros de la Mesa y el posterior debate entre éstos y el público asistente]. 
DIAGNÓSTICOS Y OPCIONES [Primer Capítulo del Tomo I] es una reflexión colectiva sobre la problemática general que hoy afronta el conocimiento histórico. Los Diagnósticos y Opciones oscilan entre la radical afirmación de la crisis - «crisis epistemológica»- en Gabrielle M. Spiegel (Towards a theory of the Midle Ground: Historical Writing in the Age of Posmodernism); la matizada negación de Santos Juliá (¿La historia en crisis?); o, para Carlos Barros (La historia que viene) -siguiendo a Tomas S. Kuhn- la especificación de la crisis como acumulación de anomalías que precede a un cambio de paradigma historiográfico. Completan este capítulo las aportaciones de Roger Chartier (L'histoire aujourd'hui: doutes, défis, propositions); Serge Deruette (Pour une «histoire causale». Plaidoyer pour une étude des causes en histoire); María Gloria Núñez (Historia, ciencia y complejidad en los finales del siglo $X X$ ); Lawrence Stone (The future of History); Gerges-Claude Tshund'Olela (Les principes de l'histoire). El recientemente fallecido Bernard Lepetit nos habla aquí sobre La societé comme un tout.

Que este capítulo se centre en el análisis sobre la/s tendencia/s historiográfica/s de conjunto, no significa ausencia de esa misma orientación en lo que resta de los contenidos de HAD. De hecho, la organización del Congreso concibió el mismo como foro de encuentro para el intercambio y profundización en la Teoría, lo que viene en parte a suponer posicionarse ante el supuesto totum revolutum de la historiografía finesecular. De este modo, los Diagnósticos y Opciones menudean a lo largo de las Actas. Pongamos como ejemplo las palabras que encabezan y las que ponen punto final a la contibución de John H. Elliot, el reciente Premio Príncipe de Asturias en Ciencias Sociales: «The state of historical writing at the end of the twentieth century is not unlike that of the world at the end of the twentieth century: fragmentation bordering on anarchy (...) cacophony of historical voices competing for attention». (Comparative history, Tomo III, pp. 9-19).

FIN DE LA HISTORIA [Capítulo Segundo, Tomo I] aborda el doble -aun opuesto- mensaje que se esconde bajo este común rótulo: finalismo histórico neohegeliano (Francis Fukuyama, por supuesto) y teoría posmoderna del fin de la historia. Contraposición y análisis de conjunto llevado a cabo por Miguel Ángel Cabrera (La historia y las teorías del fin de la historia), quien toma distancia frente a ambas caras de la moneda, 
adoptando un tono crítico que se acentúa en la intervención de Juan Manuel Santana (La historia en el fin de una época o el secuestro de Clío). Aspectos puntuales de este doble debate son tratados por Ricardo M. Martín y Guillermo Pérez (Ante el derrumbe del Muro: consideraciones sobre el totalitarismo y el socialismo real), y por Robert Muro (La idea de progreso como lastre en las filosofías de la historia: Walter Benjamin y Norberto Bobbio).

Sobre asuntos más domésticos, sobre la comunidad de los historiadores y su oficio: HISTORIOGRAFÍA, capítulo que cierra este primer tomo. Nicolai Kopossov (Vers l'anthropologie de la raison historique), Massimo Mastrogregori (Historiographie et tradition historique des souvenirs. Histoire «scientifique» des études historiques et histoire "global»du passé), Oto Luthar (The possibilities of a theory of modern historiography in changing (Eastern) Europe), dan paso al apartado específicamente dedicado a la historiografía en España (José Luis de la Granja, La historiografía española reciente), que incluye las siempre claras y entusiastas intervenciones de Ricardo García Cárcel (La manipulación de la memoria histórica) y Julio Valdeón (La historiografía española de finales del siglo XX: miseria de la teoría). Se completa el balance historiográfico con dos reflexiones en torno a problemáticas específicas vinculadas con Annales: su difusión internacional (Luis Domínguez y Xosé Ramón Santana, Renovación en la historiografía española: Antonio Eiras Roel y la recepción del movimiento Annales en Galicia), y sus singularidades (Wojciech Wrzosek, Pourquoi les Annalistes n'aiment pas la révolution [francesa]?).

Si el volumen Pasado y Futuro es una suerte de organizador previo, RETORNO DEL SUJETO [Tomo II] y OTROS ENFOQUES [Tomo III] despliegan el potencial -posibilidades y limitaciones- de los nuevos ( $g i$ ros) y de los redivivos (retornos) usos historiográficos.

RETORNO DEL SUJETO (subdividido en los capítulos: Historia de las Mentalidades, Historia Cultural, Historia de las Mujeres, Microhistoria, Actores Sociales), es decir, ampliación de la explicación en historia mediante la reapropiación de la acción-praxis, del sujeto agente, del actor -individual, colectivo-, haciendo y haciéndose (Making, recordando a E.P. Thompson), más allá de las insatisfactorias explicaciones proporcionadas por los mecanos estructural-funcionales, de muy variada especie, de muy común sesgo determinista.

"CUADERNOS DE ESTUdIOS GALLEGOS", Tomo XLV, Fascículo 110, Santiago 1998. 
HISTORIA DE LAS MENTALIDADES ofrece al lector los organizadores previos de Carlos Álvarez (La construcción social de los mundos mentales: un bricolage psicológico), Julio A. Vaquero (Mentalidades e ideologías), y Francisco Vázquez (Los problemas de la explicación en historia de las mentalidades). Se despliega a continuación el abanico temático abierto por el estudio del tercer nivel («lo mental»): Individualismo (Sophie Ollivier, Histories de l'individualisme et individualisme contemporain); Imaginario (Antonio Elorza, Imagen, religión y poder); Criminalidad y Violencia (Iñaki Bazán, La historia social de las mentalidades y la criminalidad, Doris Moreno y José Luis Beltrán, Justicia criminal y criminalidad en la Cataluña moderna: estudios y perspectivas de investigación, Ángel Rodríguez, La historia de la violencia: espacios y formas en los siglos XVI y XVII); Memoria (Paloma Aguilar, Aproximaciones teóricas y analíticas al concepto de memoria histórica. Breves reflexiones sobre la memoria histórica de la Guerra Civil Española (19361939); Muerte (Máximo García, Tendencias historiográficas recientes sobre la religiosidad popular e historia de la muerte y de las mentalidades); Documentos Personales (Alberto Angulo, «Nire Jaun eta Jabea». La expresión de las formas protocolarias en la correspondencia epistolar del setecientos).

HISTORIA CULTURAL, Capítulo Segundo del Tomo II, se abre con las contribuciones de Peter Burke (Varieties of cultural history); Robert Darnton (Diffusion vs. discourse: conceptual shifts in intellectual history and the historiography of the French Revolution); Monstse Jiménez y Javier Antón Pelayo (Propuestas metodológicas para una historia cultural de lo social. Gerona, Siglo XVIII). Al igual que el capítulo Historia de las Mentalidades, las formas de la Historia Cultural reciben tratamiento específico: Historia Intelectual (María del Mar Garrido, ¿La historia intelectual en crisis? El giro lingüístico y la historia social frente a la historia intelectual); Educación (Pere Solá, El estudio diacrónico de los fenómenos educativos y las tendencias historiográficas actuales); Literatura (Scarlett Freund, Literure and history: nonconformity and social change in Elizabeth Gaskell's 'North and South'); Historia Conceptual (Peter Oronsson, The possibilities of conceptual history «from above» and «from below»: reflections on the conceptof samhälle «society» in Sweden, 1700 to 1990); Escritura (Antonio Castillo, De la palografía a la historia. De 
las prácticas de escribir, Manuel Peña y Antoni Simón, La escritura privada en la Catalunya moderna).

HISTORIA DE LAS MUJERES [Capítulo Tercero, Tomo II] ofrece un acusado tono combativo, desde luego por completo ajeno a cualquiera neutralidad valorativa, constituyéndose en uno de los epígrafes más directamente políticos de HAD: María Luisa Bueno, Reflexiones en torno a la historia de las mujeres ¿Una historia coyuntural?; Cristina Segura, Algunas cuestiones a debatir sobre la historia de las mujeres.

MICROHISTORIA [Capítulo Cuarto, Tomo II], el estudio del «océano en una gota de agua»-según la célebre metáfora de Le Roy Ladurie-, cuenta con la aportación de James S. Amelang: Microhistory and its discontents: the view from Spain. Pese a la aparente soledad de esta contribución de Amelang, la microhistoria es referente continuo en los contenidos de Historia A Debate [por supuesto, Giovanni Levi en la Mesa C: ¿Crisis de la Historia Económica y Social?].

ACTORES SOCIALES [Capítulo Quinto, Tomo II] encara directamente la eterna y espinosa polémica sujeto-libertad vs. estructura-determinación, con la salvedad de que la reivindicada centralidad del sujeto activio pasa aquí de ser un voluntarioso enunciado humanista a configurarse como una sólida propuesta teórica [sociabilidad, explicaciones de red,...]: Giuseppe de Lucca Mobilitá sociale e richezza: le prospettive dela «Network analysis» nello estudio della Milano del XVI seculo; Francisco García, La historia de la familia o la vitalidad de la historiografía española. Nuevas perspectivas de investigación; José María Imizcoz, Actores sociales y redes de relaciones en las sociedades del Antiguo Régimen. Propuestas de análisis en historia social y política; Anaclet Pons y Justo Serna, El burgués sin atributos. Algunas reflexiones sobre el sujeto económico de la historia social.

En OTROS ENFOQUES [Tomo III] se reflexiona sobre MÉTODOS Y PROBLEMAS [Capítulo Primero] de la Historia Comparada (el ya citado John H. Elliot, Comparative History); el Tiempo (Carlos Aguirre Roja, La larga duración en el espejo (más allá del tiempo «vivido» y del tiempo «expropiado»); Julio Aróstegui, La historia como atribución. (So- 
bre el significado del tiempo histórico); la Historia Regional (Bruno Anatra, Storia locale in veste regionale: il caso italiano; Juan Pro Ruiz, Sobre el ámbito territorial de los estudios de historia); la Informática (César González Míguez, Historia e informática: las Juntas Generales de Álava; Jesús $\mathrm{M}^{\mathrm{a}}$ Usunáriz y Francisco J. Caspistegui, Un vistazo sobre algunas posibilidades de las técnicas multimedia, o de cómo convivir creativamente con un objeto llamado ordenador: el proyecto «Historia y multimedia»).

Al diálogo HISTORIA-CIENCIAS SOCIALES, a los híbridos que del mismo han brotado, se dedica el Segundo Capítulo de este Tercer Tomo, concretado en las disciplinas: Sociología Histórica (José Álvarez Junco, Aportaciones recientes de las ciencias sociales al estudio de los movimientos sociales; Custodio Velasco, Premisas conceptuales y metodológicas de «Les ouvriers en grève. France 1871-1890» de Michelle Perrot: contribución al análisis de la sociología histórica); Antropología Histórica (Yuri L. Bessmertny, L'histoire démographique anthropologiquement orientée et son devenir en Russie; André Burguière, L'anthropologie historique et l'École des Annales); Psicología e Historia (Alain Boureau, Historie et psychologie).

LOS RETORNOS [Capítulo Tercero, Tomo III] dedica su espacio a géneros y estilos (narración) antaño dominantes, aparentemente condenados al ostracismo por la historiografía del siglo XX, pero que hoy emergen con nuevos bríos. $\mathrm{Y}$ es que no es precisamente pusilaminidad lo que caracteriza a María de Fátima Bonifàcio ( $O$ abençoado retorno da vella historia), quien enfáticamente augura que $« \mathrm{O}$ renascimento da narrativa trará consigo o renascimento da historia, nâo como Ciência Social, mas como disciplina literaria» [p. 155, énfasis en el original], afirmación a la que llega a través de una filípica contra la sombra de una reduccionista caricatura epistemológica, según la cual ciencia es, para la autora, sinónimo del más burdo y malévolo objetivismo de la verificación -ni tan siquiera la falsabilidad tiene cabida dentro de este batallar contra molinos de viento-, ánimo exorcista que se ejerce con especial saña sobre el maridaje historia-ciencais sociales: «imposiçao duma disciplina conceptual importada das Ciências Sociais e servida por un arsenal de termos técnicos destinados a impressionar o leitor» [p. 153, el énfasis es

"CUADERNOS DE ESTUDIOS GALLEGOS", Tomo XLV, Fascículo 110, Santiago 1998. 
nuestro]. En definitiva, un subproducto más de dadaísmo epistemológicoteórico, que, entre otras licencias, se permite, por una parte, lastrar al enemigo creado con una muy poco informada conceptuación de ciencia -la de un intrasubjetivismo, digamos, absoluto-, para, por otra parte, reclamar para sí una lejana -muy lejana- aproximación a más intersubjetivistas, consensualistas,... más humanistas nociones (ya elaboradas: Lakatos, Kuhn) de ciencia, dado que Bonifàcio dadivosamente concede a la historia «uma reserva capital, que a distingue de outras formas de arte: tem de ser uma opiniâo informada (...) sobre o mundo», [p. 156, el primer énfasis es nuestro] ${ }^{1}$.

Les retours dans l'historiographie française actuelle es la aportación de Jacques Le Goff. Ubicada tras el artículo de Bonifàcio, puede ser leída a modo de antídoto contra el aguijoneo de la «vieja historia». Confirma el historiador francés «toute conception historiographique doit, me sembletil, recourir épisodiquement à des séquences narratives mais évidemment ce n'est pas que l'on peut appeler de l'histoire-récit. (...) importe de rendre compte que l'histoire-récit n'est pas plus innocente que les autres formes d'histoire, elle procéde aussi du faire de l'histoire, d'operations de montages de l'histoire dont le résultat est d'autant plus redoutable que le no-dit qu'elle suppose échappe au consommateur est probablement l'historie la plus inconsceimment idélogique» [p. 161].

Más allá de numantinas reivindicaciones a favor de un «eterno retorno de lo idéntico», se interrogan los historiadores sobre los matices -rigor analítico contra desbordada floración de la $d o x a$ - que sugiere este rejuvenecer de los viejos-nuevos géneros historiográficos: ¿narración $=$ nocientificidad?; ¿puede la biografía individual contribuir a explicar al biografía colectiva?; ¿qué relaciones establecer entre lo político, lo militar y lo económico-social? Un largo etcétera de cuestiones abiertas al debate. También retorno, pues, a la historia-problema (Febvre) en los cuatro epígrafes que subdividen el capítulo dedicado a Los Retornos: Biografía Histórica (Francisca Colomer, Biografía y cambio social: la historia que

\footnotetext{
' Si lo que se busca es un análisis no realizado a martillazos, consúltese al respecto Tomas S. Kuhn: «Comentarios sobre las relaciones de la ciencia con el arte (La tensión esencial. Estudios selectos sobre la tradición y el cambio en el ámbito de la ciencia, Fondo de Cultura Económica, Madrid, 1993, pp. 365-377).
}

"CUADERNOS DE ESTUDIOS GALLEGOS", Tomo XLV, Fascículo 110, Santiago 1998. 
estamos viviendo, Susana Strozzi, Sujeto y persona en la biografía histórica); Historia Narrativa (Antonio Morales Moya, Paul Ricoeur y la narración histórica); Historia Política (Xabier Gil Pujol, La historia política de la Edad Moderna europea, hoy: progresos y minimalismo); Christophe Prochasson, Vingt ans d'histoire politique sans l'État: mutations et reformulations, José J. Ruiz Ibáñez, Sobre la crisis de 1590: no Historia Política, sino historia hecha con materiales documentales y procesos de análisis políticos); Historia Militar (Antonio Espino López, La historia política y la renovación de la historia militar).

Con LA HISTORIA ENSEÑADA [Capítulo Cuarto, Tomo III] llegamos al final de nuestro recorrido por HAD. Y llegamos, también, a la certeza del tópico: «por último no menos importante». Pues, ¿para qué, para quién,... el conocimiento histórico?, pragmáticos interrogantes que no sólo interesa responder a la comunidad -corporación preferirán decir algunos- de los historiadores, cuestiones que afectan a disciplinas fronterizas como la Teoría Política, «que aunque ha evitado en gran medida el solipsismo sobre el que solían cerrarse muchas tradiciones teóricas locales (...), no ha sabido evitar muchas veces un cierto apartamiento de la política concreta, ni el refugio en una jerga esotérica solamente accesible a los ya iniciados. Sin tener por qué perder el necesario rigor académico, nuestra asignatura pendiente puede que resida, después de todo, en buscar una mayor proximidad a nuestro verdadero objeto: la política [vale decir la historia] 'real'», (Vallespín, F., «La teoría política hoy», in idem. (ed.), Historia de la Teoría Política/6, Alianza, Madrid, 1995, p. 13).

Sobre superación del solipsimo-esoterismo, sobre conexión del historiador con la política, la sociedad, la historia «reales» a través -y más allá- de la divulgación institucionalizada, nos habla LA HISTORIA ENSEÑADA en la Universidad (Margarita Aizpuru, Adolfo Borrero, José García, Francisco Melero y Carmen Rocío, La universidad más allá de la institución. La historia más allá de la universidad), y en la Enseñanza Media (José Manuel Navarro, La concepción de la mujer a mediados del s. XIX: una experiencia de investigación en el aula. Una forma distinta de enseñar historia; $\mathrm{M}^{\mathrm{a}}$ Josefa Parejo y Adela Tarifa, Nuevos enfoques para la didáctica de la historia en la enseñanza secundaria: hacia una metodología interdisciplinar y participativa; Julio Prada, Historia y reforma. Una reflexión crítica en torno a la estructuración curricular por 
áreas y el recurso al entorno como instrumento pedagógico; Ángel Trigueros, El concepto de historia en las enseñanzas medias. La función educativa y la función social de la historia).

Tras la apretada descripción de tan denso índice, parece conveniente intentar la búsqueda de algunas conclusiones, acotar puntos de referencia sobre un fondo común de los historiadores que, aparentemente, más se asemeja al desasosiego de Pollock y su expresionismo abstracto que a una plácida cuadrícula more geométrico-cartesiano. Pero incluso el dripping atiende a los principios de una composición. Intentemos la composición de lugar que Historia A Debate nos sugiere:

- HAD muestra cómo el historiador, si es que alguna duda cabía tras el desarrollo historiográfico del siglo XX, se afirma como teórico, gestiona su propio utillaje conceptual, $\mathrm{y}$, complementariamente, somete a examen -asimila o rechaza- las propuestas de la teoría económica, la teoría social, la lingüística,... la filosofía. Empeño teoricista que no es un superfluo barniz retórico artificiosamente impuesto por encima de una supuestamente más básica investigación empírica, positiva, máxime cuando lo que hoy está en la base de la alternativa teórica posmoderna es que «su aceptación acrítica privaría a la historia y a las demás ciencias sociales de su razón de ser»; de ahí «La imperiosa necesidad de que la investigación y la profesión históricas se incorporen plenamente al debate suscitado por el pensamiento posmoderno y por su teoría del fin de la historia, pues en dicho debate se decide en gran medida el perfil futuro de la investigación social», (Miguel Ángel Cabrera Acosta, La historia y las teorías del fin de la historia, Tomo I, p. 220).

- Si HAD explicita una dispersión temática evidentemente en alza, ello no debería servir como coartada para velar la permanencia o reafirmación de consensos elementales pero de inmensa relevancia: se percibe una orientación general hacia la explicación compleja, relacional -por evitar el retrogusto metafísico de los términos total, totalidad-, en un doble sentido: $1^{\circ}$. reconocer la complejidad de interrelaciones entre niveles (lo económico-lo social-lo político-lo mental...), es decir, no se renuncia a la siempre retóricamente invocada histoire globale, durante largo tiempo encorsetada en su rígido marco [macro]estructura; $2^{\circ}$. reconocer la insuficiencia de los enfoques macroestructurales para abordar la explicación de la reproducción-estabilidad y el cambio sociales en la 
historia, impasse del cual sólo hemos podido comenzar a salir una vez que hemos percibido la fertilidad de los enfoques micro (microhistoria, redes de actores sociales,...), que no son una herejía más, una moda más en el turbio y amorfo fango de la posmodernidad ambiente. Buena prueba de ello es que no sólo a los historiadores les vienen carcomiendo esas insuficiencias, esas anomalías. El marxismo analítico reacciona contra ellas proponiendo «explicar instituciones y procesos sociales en términos de las acciones y relaciones entre los individuos. En definitiva, la idea es buscar los microfundamentos, las tuercas y tornillos de aquello que se pretende explicar», (Gargarella, R., «Marxismo Analítico», Claves de Razón Práctica, 62 (Mayo 1966), p. 62).

- Con la excepción de algún que otro francotirador, los retornos no plantean una incorrupta resurrección de la Vieja Historia. En términos generales, las intervenciones en HAD sobre esta cuestión muestran la conveniencia de enganchar lo biográfico, la narrativa -no el relato novelado-, lo político -no la política/institución-, lo militar,... con los -insuficientes, pero no desechables- desarrollos historiográficos del siglo XX (Historia Económica y Social,... Historia Relacional), siguiendo los retornos más la trayectoria de una espiral ascendente que la de un círculo-circuito cerrado. Un ejemplo significativo: incluso la otrora vergonzante historia militar (histoire-bataille), la denostada historia de «trompetas y tambores», gira hasta derivar -no sin antes haber edificado «una 'socialización' de la historia militar» (A. Espino, La historia política y la renovación de la historia militar, Tomo III, p. 248)- hacia la problemática del sujeto: «reconstrucción de la batalla desde el punto de vista de los participantes (...) superación de la historia batalla al tratar del soldado en la batalla», (idem. p. 251, énfasis en el original). ¿Y no es también ésta una variante más de la historia desde abajo? ¿No es ésta una aportación más a los enfoques micro?

Por lo demás, los retornos tienen múltiples aristas: ¿no supuso -o todavía está suponiendo- la emergencia de la Historia de las Mentalidades un retorno a Febvre (Le problème de l'incroyance au l6e siècle: la religion de Rabelais), Bloch (Les rois thaumaturges) o Lefebvre (La grande peur de 1789), tras el prolongado paréntesis de la hiperestructuralista historia braudeliana? La respuesta nos la sirve Peter Burke: «Junto con Los reyes taumaturgos de Bloch y el artículo de Lefebvre sobre las multitudes, este trabajo [de Febvre, El problema de la incredulidad...] inspiró 
la historia de las mentalidades colectivas a la que tantos historiadores franceses se entregaron a partir de 1960», (La revolución historiográfica francesa. La Escuela de los Annales 1929-1984, Gedisa, Barcelona, 1993, p. 34).

- Coherencia tras un desmigajamiento aparente, consensos -conscientemente asumidos o inadvertidamente operativos- ya en acto o que comienzan a perfilarse, no implican diálogo de guante blanco. No son cuestiones de mínimo retoque lo que se debate en Final de la Historia, Historia de las Mujeres,... Mantener el debate en los límites de cortes analíticos discretos (ontología / epistemología / metodología / teoría...) nos permite no caer en el descontrol cognitivo, nos impide hacer trampa (p.e. identificar totalidad en el metarrelato ilustrado con totalidad como constructo teórico en la ciencia social). Pero ingenuo sería creer que ese respeto hacia las reglas del juego analítico, ese endiosamiento del método, nos estuviera mecánicamente vehiculando hacia la solución de la crisis hisțoriográfica. En HAD se abunda en la dimensión crítica, y, en último término, política, individual y socialmente no neutral, del conocimiento histórico. De entre todas las manifestaciones al respecto sirvan de muestra -y concluimos- las palabras del editor: «O la humanidad devuelve al hombre, y a su medio ambiente, al centro de interés de la actividad política y económica, o el descalabro final -económico, ecológico, demográfico, ético, social- a manos de la tercera revolución tecnológica y de la prepotencia del Primer Mundo, está asegurado. La historia y las ciencias humanas tienen algo que decir, y van a decirlo, siempre y cuando el paradigma historiográfico culmine satisfactoriamente el cambio en curso, que no tiene meta pre-establecida: depende de nosotros», (Carlos Barros, $\mathrm{La}$ historia que viene, Tomo I, p. 117).

"CUADERNOS DE ESTUDIOS GALLEGOS", Tomo XLV, Fascículo 110, Santiago 1998. 